Conference Article

\title{
Investigating the Nonlinear Dynamics of Emerging and Developed Stock Markets
}

\section{K. Guhathakurta}

\author{
Finance \& Accounting Indian Institute of Management Indore,Prabandh Shikhar, Rau-Pithampur RoadIndore, Madhya Pradesh, \\ India -453356
}

\begin{abstract}
Financial time-series has been of interest of many statisticians and financial experts. Understanding the characteristic features of a financial-time series has posed some difficulties because of its quasi-periodic nature. Linear statistics can be applied to a periodic time series, but since financial time series is non-linear and non-stationary, analysis of its quasi periodic characteristics is not entirely possible with linear statistics. Thus, the study of financial series of stock market still remains a complex task having its specific requirements. In this paper keeping in mind the recent trends and developments in financial time series studies, we want to establish if there is any significant relationship existing between trading behavior of developing and developed markets. The study is conducted to draw conclusions on similarity or differences between developing economies, developed economies, developing-developed economy pairs. We take the leading stock market indices dataset for the past 15 years in those markets to conduct the study. First we have drawn probability distribution of the dataset to see if any graphical similarity exists. Then we perform quantitative techniques to test certain hypotheses. Then we proceed to implement the Ensemble Empirical Mode Distribution technique to draw out amplitude and phase of movement of index value each data set to compare at granular level of detail. Our findings lead us to conclude that the nonlinear dynamics of emerging markets and developed markets are not significantly different. This could mean that increasing cross market trading and involvement of global investment has resulted in narrowing the gap between emerging and developed markets. From nonlinear dynamics perspective we find no reason to distinguish markets into emerging and developed any more.
\end{abstract}

Keywords: Empirical Mode Decomposition, Emerging Market ,Nonlinear Dynamics

\section{Introduction}

Financial time-series has been of interest of many statisticians and financial experts. Understanding the characteristic features of a financial-time series has posed some difficulties because of its quasi-periodic nature. Linear statistics can be applied to a periodic time series, but since financial time series is non-linear and non-stationary, analysis of its quasi periodic characteristics is not entirely possible with linear statistics. Thus, the study of financial series of stock market still remains a complex task having its specific requirements.

The study of stock price movements commenced long time back. Bachelier [1] was first to formulate theory of random walk hypothesis. He showed that empirical evidence confirmed the random walk hypothesis i.e. a series of price changes are memory less, they follow a Markov process. This evidence confirms the efficient market hypothesis (EMH). Efficient market hypothesis states that the current price reflects all the available information about the security. Thus, predicting a price change is not possible, unless new

* E-mail address: kousikg@gmail.com

ISSN: 1791-2377 @ 2015 Kavala Institute of Technology. All rights reserved. information comes in. EMH has been received with much skepticism, and still the use of charts and volume data are rampant in predicting stock price movements. The main argument in disproving the effectiveness of technical analysis is the use of auto correlations. But, the use of such linear statistics may not be able to capture the complexities of non-linear patterns time series exhibits. As stated by Campbell et al. [2], "several authors signal a growing interest in technical analysis among financial academics, and so, it may become a more active research area in the near future".

Many important works have been done in this domain. For example, Dieci et al. (3) modeled the relationship between moods, beliefs and asset price. Similarly a behavioral model of asset price was worked out by Chiarella et al. (4). Pezzo and Uberti (5) worked on models for emerging equity markets. Hiller - Band and Weinzelburger (6) produced some interesting work on multi period capital asset pricing model. Marseguerra et al. (7) worked on investment timing decisions. Bo and Quingxin (8) derived the boundary prices for American contingent claims in a general continuous time market model with taking into account transaction prices. Ahmed and Abdusalam (9) proposed a modified BS equation that corresponds to telegraph equation. Tang et al. [10] addressed the hedging 
problem of American Contingents Claims (ACCs) in the framework of continuous-time Ito ${ }^{\wedge}$ models for financial markets. Cajuerio and Tabak [11] worked on relative efficiency and suggested that Asian equity markets showed greater inefficiency than those of Latin America (with the exception of Chile), and that developed markets ranked first in terms of efficiency. Batten et al. [12] investigated the sensitivity of the long-term return anomaly observed on the Nikkei stock index to sample and method bias. Strozzi and Comenges [13] proposed a new trading strategy based on state space reconstruction techniques.

In this paper keeping in mind the recent trends and developments in financial time series studies, we want to establish if there is any significant relationship existing between trading behavior of developing and developed markets. We then apply a new technique for analysing the periodicity and properties of time series. Many "paradoxes" exist in standard decomposition of time signals. To avoid them, Huang et al. (1998)[14] have developed a method, termed the Hilbert view, for studying non-stationary and nonlinear data in nonlinear dynamics. This method uses the empirical mode decomposition technique to generate finite number of Intrinsic Mode Functions (IMFs) that assume well behaved Huang-Hilbert Transform [14)]. The results obtained from this technique can be further analysed to reveal more details about the time series. Guhathakurta et al. (2008) [15] uses this technique to a good effect to compare two financial time series.

The study is conducted to draw conclusions on similarity or differences between developing economies, developed economies, developing-developed economy pairs. We take the leading stock market indices dataset for the past 15 years in those markets to conduct the study. First we have drawn probability distribution of the dataset to see if any graphical similarity exists. Then we perform quantitative techniques to test certain hypotheses. Then we proceed to implement the Ensemble Empirical Mode Distribution (EEMD) technique to draw out amplitude and phase of movement of index value each data set to compare at granular level of detail. The EEMD technique was developed by $\mathrm{Wu}$ and Huang (2009)[16]. This new approach consists of sifting an ensemble of white noise-added signal and treats the mean as the final true result. The effect of the added white noise is to provide a uniform reference frame in the time-frequency space; therefore, the added noise collates the portion of the signal of comparable scale in one IMF. The rest of the paper is arranged in the following way- section 2 gives details about the data followed by section 3 elaborating the methodology and approach. We present the analysis of the results in section 4 followed by the conclusions in section 5 .

\section{Data}

The series studied in this analysis include nine emerging stock market indices, BUX (Hungary), CSI 300(China), IBOVESPA (Brazil), IPSA (Chile), KLSE (Malaysia), KOSPI (Korea), MXX-IPC (Mexico), S\&P CNX Nifty (India) and TWII (Taiwan) and seven developed stock market indices, AORD (Australia), DAX (Germany), FCHI (France), FTSE 100 (UK), HANGSENG (Hong Kong), NIKKEI (Japan), NZE 50 (New Zealand) at daily frequencies. The market classification into developed and emerging is based on Morgan Stanley Capital International (MSCI). The MSCI market classification scheme depends on the following three criteria: economic development, size and liquidity, and market accessibility. A market is classified as developed if: i) the country's Gross National Income per capita is $25 \%$ above the World Bank high income threshold for 3 consecutive years; ii) there is a minimum number of companies satisfying minimum size and liquidity requirements; and iii) there is a high openness to foreign ownership, ease of capital inflows/outflows, high efficiency of the operational framework and stability of the institutional framework. To be included in the emerging market category, a market is characterized by size, liquidity and market accessibility criteria that are less tight than those for the developed markets.

\section{Design, Methodology and Approach}

The approach can be summarized in the following steps:

- Find out the IMF's of the given market returns using Empirical Mode Decomposition

- Apply Hilbert's transform to the IMF's to get the instantaneous phase and amplitudes

- Plot the probability distributions of phase and amplitudes of IMF's

- Compare the probability distributions calculated above of various market indices

The first step is find out the IMF's of various returns using EMD Empirical Mode Decomposition (EMD) is designed primarily for obtaining representations which are oscillatory, possibly non stationary or generated by a nonlinear system, in some automatic, fully data-driven way. We first decomposed the data into slow and high frequency parts. The slow frequency part was again decomposed into further slow and high frequency parts. The process was continued till series was deemed to be as fully decomposed.

An IMF is defined as a function that satisfies the following requirements:

1. In the whole data set, the number of extrema and the number of zero-crossings must either be equal or differ at most by one.

2. At any point, the mean value of the envelope defined by the local maxima and the envelope defined by the local minima is zero

The procedure of extracting an IMF is called sifting. The sifting process is as follows:

1. Identify all the local extrema in the test data.

2. Connect all the local maxima by interpolation as the upper envelope.

3. Repeat the procedure for the local minima to produce the lower envelope.

The upper and lower envelopes should cover all the data between them. Their mean is $m_{1}$. The difference between the data and $\mathrm{m}_{1}$ is the first component $\mathrm{h}_{1}$ :

$X(t)-m_{1}=h_{1}$

Ideally, $\mathrm{h}_{1}$ should satisfy the definition of an IMF, for the construction of $h_{1}$ described above should have made it symmetric and having all maxima positive and all minima negative. After the first round of sifting, a crest may become a local maximum. New extrema generated in 
this way actually reveal the proper modes lost in the initial examination. In the subsequent sifting process, $\mathrm{h}_{1}$ can only be treated as a proto-IMF. In the next step, it is treated as the data, then

$h_{1}-m_{11}=h_{11}$

After repeated sifting up to $\mathrm{k}$ times, $\mathrm{h}_{1}$ becomes an IMF, that is

$h_{1(k-1)}-m_{1 k}=h_{1 k}$ the data:

Then, it is designated as the first IMF component from

$c_{1}=h_{1 k}$

Overall, $\mathrm{c}_{1}$ should contain the finest scale or the shortest period component of the signal. We can, then, separate $\mathrm{c}_{1}$ from the rest of the data by $X(t)-c_{1}=r_{1}$. Since the residue, $r_{1}$, still contains longer period variations in the data, it is treated as the new data and subjected to the same sifting process as described above.

This procedure can be repeated to all the subsequent $r_{j}$ 's, and the result is

$r_{n-1}-c_{n}=r_{n}$

The sifting process stops finally when the residue, $r_{n}$, becomes a monotonic function from which no more IMF can be extracted. From the above equations, we can induce that

$X(t)=\sum_{j=1}^{n} c_{j}+r_{n}$

Thus, a decomposition of the data into n-empirical modes is achieved.

A Hilbert-transform can be applied to an IMF to get the instantaneous phase and amplitudes. To get the Hilbert transform on each IMF component, the original data can be expressed as the real part, Real, in the following form:

$X(t)=\operatorname{Real} \sum_{j=1}^{n} a_{j}(t) e^{i f \omega_{j}(t) d t}$

The probability distributions of instantaneous phase and amplitudes of the phase and amplitudes is then calculated for each IMF's and compared across the stock market incides.

\section{Analysis and Findings}

Looking at the Empirical Mode Decompositions of the respective data sets representing the different time series (Appendix-1) we do not find any significant difference between them. If we look at the number of IMFs which is an indication of the degree of nonlinearity in the data (Appendix-1), we find that the number hovers between 11 and 12, while for the developing countries; it is also between 11 and 12 . In case of emerging markets we expected a lot more nonlinearity and therefore, a significant increase in the number of constituent IMFs. However, we find that is not the case.

We looked at the distribution of the IMF values and found that while there are differences between countries there is no significant difference between emerging and developed countries. This is evident once we analyse the correlation matrix for the IMF values of the Empirical Mode Decompositions of the respective stock index time series of the Developed and Emerging countries. (Appendix-2). We can see that the correlation of the $1^{\text {st }}$ IMF values between the Developed countries and those between the Emerging countries are not significantly different from those between the Emerging and developed countries). In the same way, we compared the results of $2^{\text {nd }}$ IMF and came to the same conclusion as evident from Table 1, 2 \& 3 of Appendix-2. This clearly indicates that the dynamics as revealed by the empirical mode decomposition is not very dissimilar in case of developed and emerging countries.

To confirm our findings we also computed the frequency distribution of the instantaneous phase and amplitude of the $1^{\text {st }}$ IMF $\mathrm{s}$ of the different time series under study. These would reveal the difference in characteristics of the dynamics of the data series at a finer level. If the time series exhibit entirely different characteristics in terms of nonlinearity then the frequency distribution of two different IMF phase and amplitude will be entirely different from each other. We expect to discover major differences between the emerging and developed data sets at least. Looking at the frequency distribution of $1^{\text {st }}$ IMF $s$ of the empirical mode decomposition of the different stock index time series (Appendix 3, Fig 6-8), we do not find any significant difference between the two sets of data. We find that the distributions follow similar pattern in case of both the developed and emerging market data. If we further extend our analysis to the distribution of the instantaneous phases of the different stock market data (Appendix 4, Fig 9-12), we find the same picture. We cannot significantly distinguish between emerging and developed country data. We find that the essential dynamics as revealed by empirical mode decomposition are same for developed and so called emerging markets.

\section{Conclusions and Research Implications}

Empirical Mode Decomposition is a method that helps decompose a time series into several constituent series, which, if added, will lead to the original time series. The number and nature of such constituent series called the intrinsic mode function (IMF) depend on the nonlinear dynamic properties of the original data set itself. Thus by looking at the number and properties of such IMFs one can compare two or more time series effectively. If the time series have similar nonlinear dynamic properties the number and nature of the IMFs will also be approximately same. We use this property of EMD to good effect by studying different financial time series from multiple markets grouped under developed and emerging markets. Our findings lead us to conclude that the nonlinear dynamics of emerging markets and developed markets are not significantly different. This could mean that with increasing cross market trading and involvement of global investment has resulted in narrowing the gap between emerging and developed markets. From nonlinear dynamics perspective we find no reason to distinguish markets into emerging and developed any more. In this respect it is interesting to note that the findings of Guhathakurta et al. ( 2012)[18], which tested the long memory properties of the same markets also came to similar conclusions in terms of memory characteristics of the two different market groups. 
Further studies may look at other investigations like the phase space evolution and recurrence analysis of the time series to try and find out whether it is time to conclude that there is essentially no difference between developed and emerging stock markets any more.

\section{References}

[1] Bachelier L, Le jeu, la chance, et le hasard Flammarion. Paris; 1914.

[2] Campbell J, Lo A, MacKinley A. The econometrics of financial markets. Princeton University Press: Princeton University Press; 1997.

[3] Dieci R, Forona I, Gardini L, He Xue-Zhong. Chaos, Solitons \& Fractals 2006; 29:520.

[4] Chiarella C, He Xue-Zhong, Wang Duo. Chaos, Solitons \& Fractals 2006; 29:535.

[5] Pezzo R, Uberti M. Chaos, Solitons \& Fractals 2006; 29:556.

[6] Hillebrand M, Wenzelburger J. Chaos, Solitons \& Fractals 2006; 29:578.

[7] Marseguerra G, Cortelezzi F, Dominioni A. Chaos, Solitons \& Fractals 2006;29:611.

[8] Bo Wang, Qingxin Meng. Chaos, Solitons \& Fractals 2005; 23:1153.

[9] Ahmed E, Abdusalam HA. Chaos, Solitons \& Fractals 2004; 22:583.

[10] Tang Maoning, Qingxin Meng, Bo Wang. Chaos, Solitons \& Fractals 2007; 31:269.
[11] Cajueiro D, Tabak M. Chaos, Solitons \& Fractals 2004; 22:349.

[12] Batten JA, Ellis C, A Fetherston T. Chaos, Solitons \& Fractals $2005 ; 23: 1125$.

[13] Strozzi F, Comenges Jos-Manuel Zaldvar. Chaos, Solitons \& Fractals 2006; 28:601.

[14] Huang NE, Shen Z, Long SR, Wu MC, Shih HH, Zheng Q, et al. Proc R Soc London Ser A 1998;454:903.

[15] Guhathakurta, Kousik, Indranil Mukherjee, and A. Roy Chowdhury. "Empirical mode decomposition analysis of two different financial time series and their comparison." Chaos, Solitons \& Fractals 37.4 (2008): 1214-1227.

[16] Wu, Zhaohua, and Norden E. Huang. "Ensemble empirical mode decomposition: a noise-assisted data analysis method." Advances in Adaptive Data Analysis 1.01 (2009): 1-41.

[17]Guhathakurta Kousik, Bhattacharya SN, Bhattacharya M, IIMK working paper no IIMK/WPS/107/FIN/2012/10 
APPENDIX 1: Empirical Mode decomposition of some of the stock market data

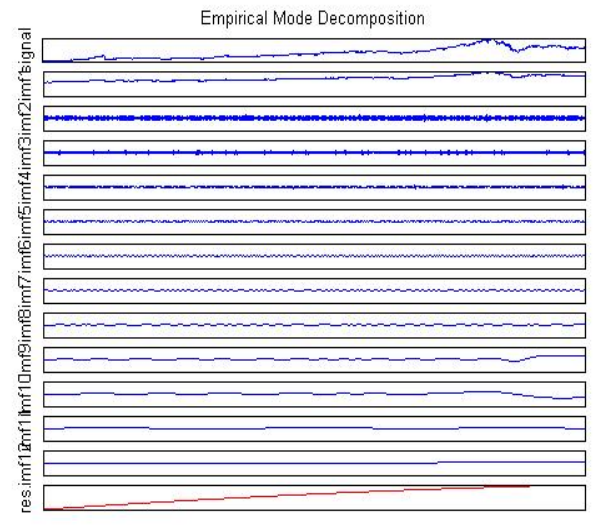

Fig 1 Empirical Mode decomposition of AORD

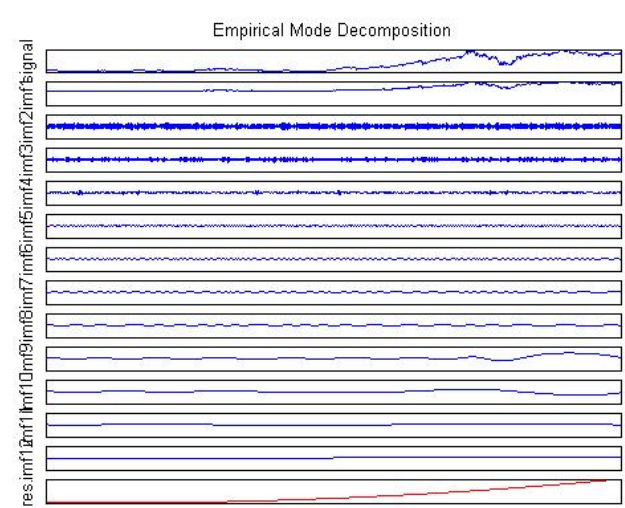

Fig 3 Empirical Mode decomposition of NIFTY

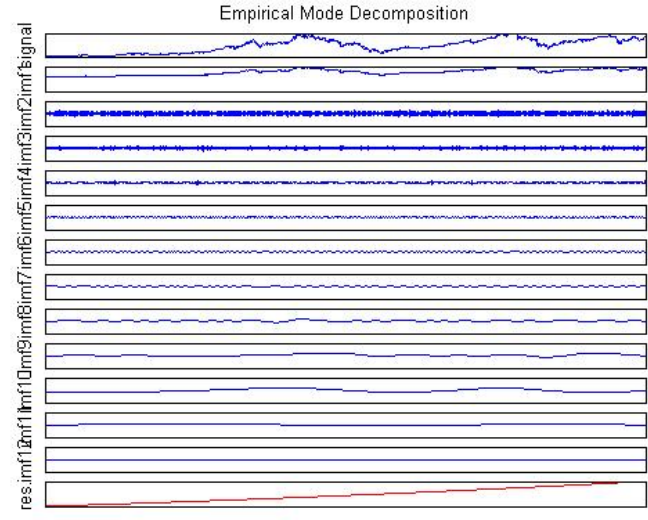

Fig 2 Empirical Mode decomposition of DAX

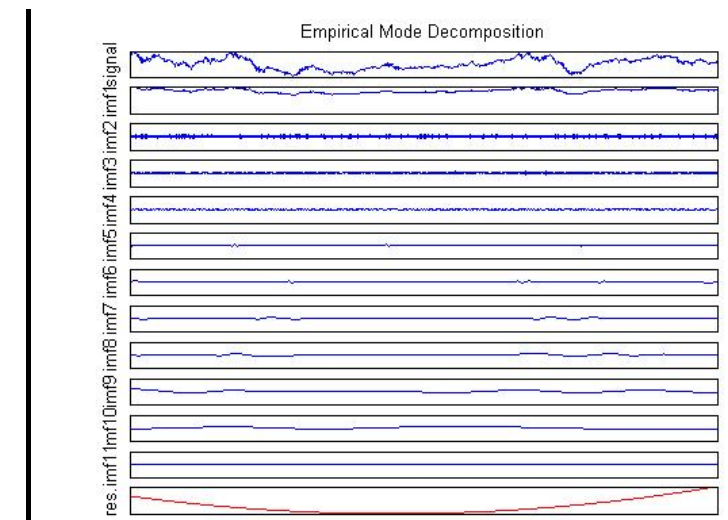

Fig 4 Empirical Mode decomposition of TWII

Appendix 2: Correlation between $1^{\text {st }} \& 2^{\text {nd }}$ IMFs

Table 1: Correlation between $2^{\text {nd }}$ IMFs of Emerging Country data

\begin{tabular}{|c|c|c|c|c|c|c|c|c|c|}
\hline & BUX & CSI & IBOVESPA & IPSA & KLSE & KOSPI & MXX & NIFTY & TWII \\
\hline BUX & 1.000 & 0.076 & -0.022 & -0.026 & 0.056 & 0.010 & -0.011 & -0.029 & 0.014 \\
\hline CSI & & 1.000 & 0.018 & 0.003 & -0.004 & 0.071 & -0.096 & -0.020 & 0.062 \\
\hline IBOVESPA & & & 1.000 & -0.040 & 0.023 & -0.066 & -0.027 & 0.013 & 0.026 \\
\hline IPSA & & & & 1.000 & -0.006 & 0.006 & -0.012 & -0.042 & 0.053 \\
\hline KLSE & & & & & 1.000 & 0.014 & 0.003 & -0.065 & 0.015 \\
\hline KOSPI & & & & & & 1.000 & -0.052 & -0.039 & 0.001 \\
\hline MXX & & & & & & & 1.000 & 0.026 & 0.017 \\
\hline NIFTY & & & & & & & & 1.000 & -0.016 \\
\hline TWII & & & & & & & & - & 1.000 \\
\hline
\end{tabular}

Table 2: Correlation between $2^{\text {nd }}$ IMFs of Developed country data

\begin{tabular}{|c|c|c|c|c|c|c|c|}
\hline & AORD & DAX & FCHI & FTSE & Hangseng & Nikkei & NZX \\
\hline AORD & 1.000 & 0.027 & -0.004 & -0.003 & -0.001 & -0.011 & -0.013 \\
\hline DAX & & 1.000 & -0.013 & -0.026 & -0.006 & -0.021 & 0.031 \\
\hline FCHI & & & 1.000 & 0.000 & 0.030 & -0.016 & -0.012 \\
\hline FTSE & & & & 1.000 & -0.005 & 0.015 & -0.043 \\
\hline Hangseng & & & & & 1.000 & -0.009 & 0.036 \\
\hline Nikkei & & & & & & 1.000 & -0.025 \\
\hline NZX & & & & & & & 1.000 \\
\hline
\end{tabular}


Table 3: Correlation between 1st IMFs of Developed \& Emerging country data

$\begin{array}{llllllll} & \text { AORD } & \text { DAX } & \text { FCHI } & \text { FTSE } & \text { Hangseng } & \text { Nikkei } & \text { NZX } \\ \text { BUX } & 0.009 & -0.017 & -0.044 & 0.000 & 0.016 & -0.024 & 0.025 \\ \text { CSI } & -0.023 & -0.053 & 0.014 & -0.047 & 0.001 & 0.021 & 0.009 \\ \text { IBOVESPA } & 0.004 & 0.008 & 0.019 & 0.003 & -0.001 & 0.022 & 0.015 \\ \text { IPSA } & -0.019 & -0.007 & 0.004 & -0.011 & 0.024 & 0.028 & 0.033 \\ \text { KLSE } & 0.005 & 0.030 & 0.004 & -0.032 & 0.033 & 0.024 & 0.019 \\ \text { KOSPI } & -0.008 & 0.002 & -0.021 & -0.018 & 0.017 & 0.000 & 0.011 \\ \text { MXX } & -0.001 & -0.011 & 0.014 & -0.007 & 0.019 & 0.012 & -0.026 \\ \text { NIFTY } & 0.011 & 0.014 & 0.005 & 0.012 & -0.001 & -0.002 & 0.040 \\ \text { TWAII } & -0.020 & 0.008 & 0.027 & 0.023 & 0.002 & 0.017 & -0.007\end{array}$

Appendix 3: Frequency distribution of instantaneous amplitude of $1^{\text {st }}$ IMF of some stock markets
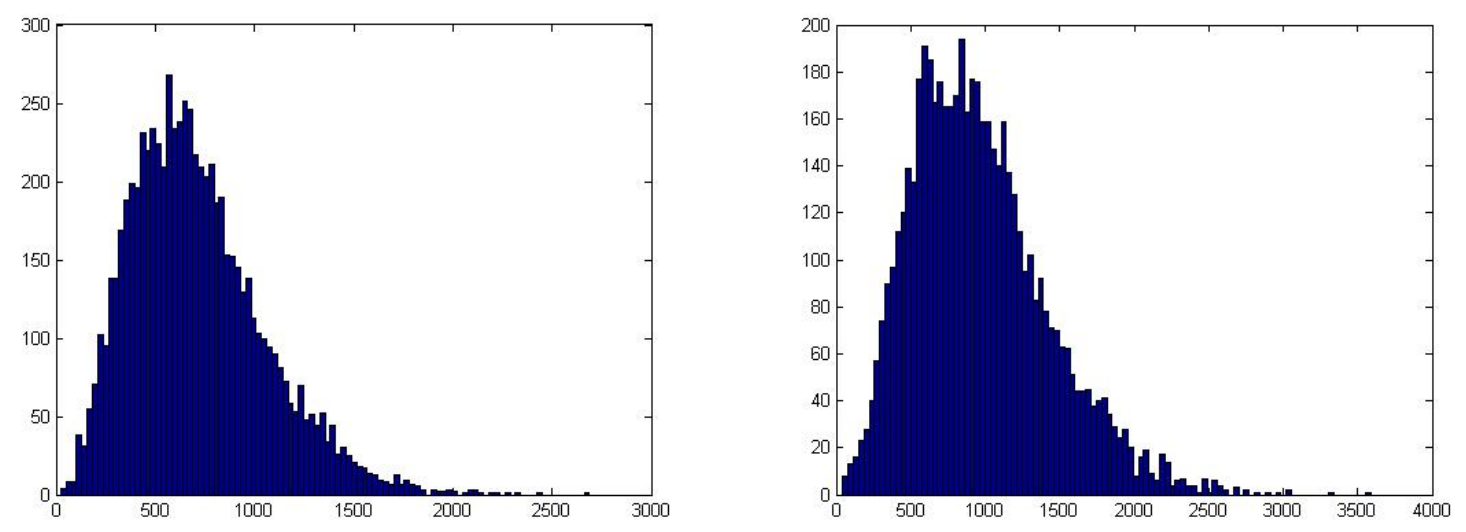

Fig 5 AORD (Australia)

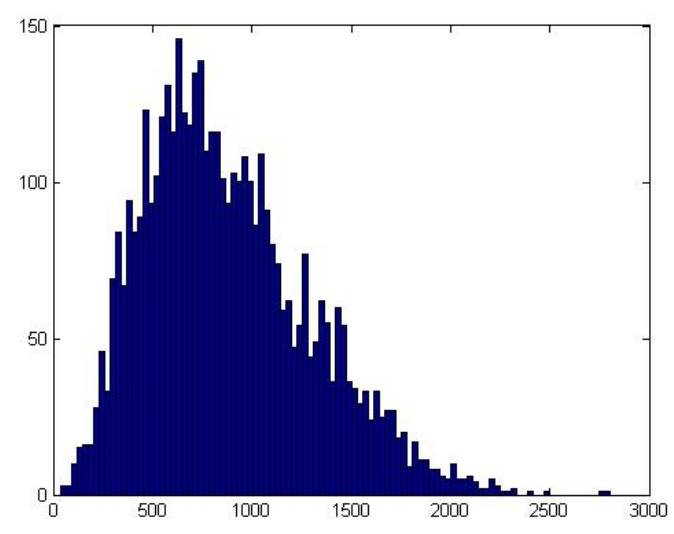

Fig 6 DAX (Germany), DJA (USA),
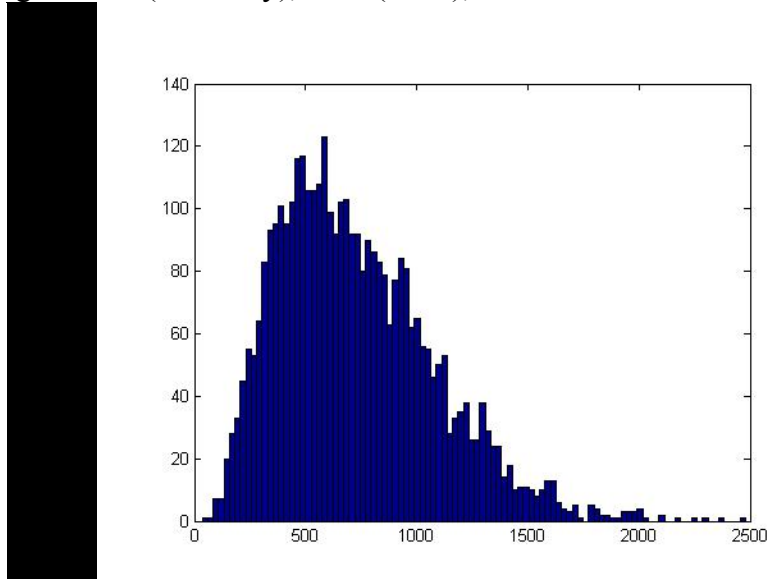

Fig 7 NIFTY

Fig 8 TWII 
Appendix 4: Frequency distribution of instantaneous phase of $1^{\text {st }}$ IMF

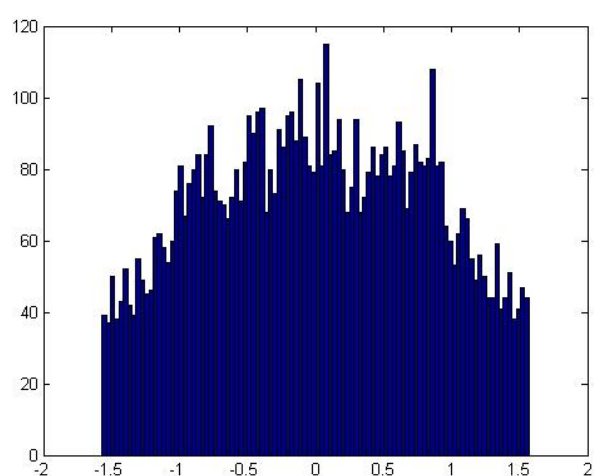

Fig 9 AORD

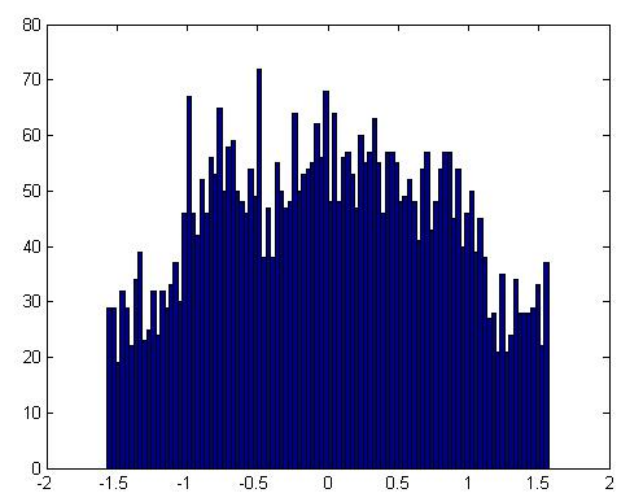

Fig 11 NIFTY
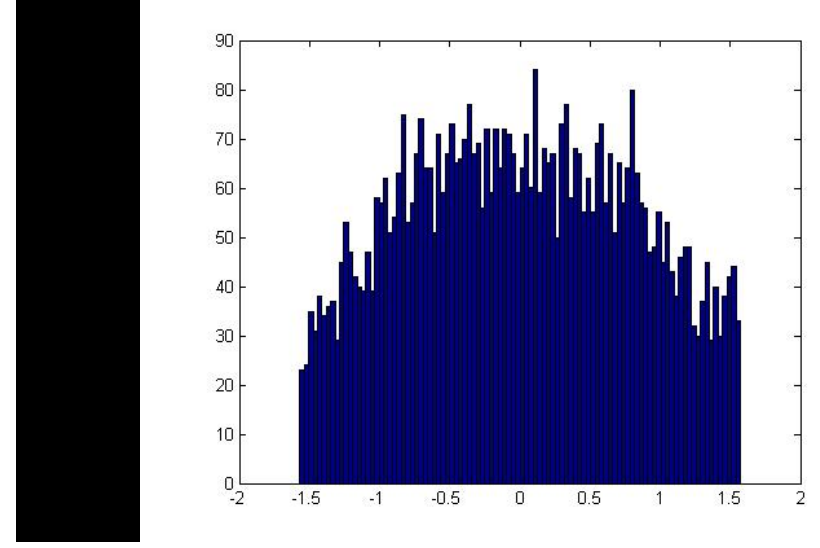

Fig 10 DAX

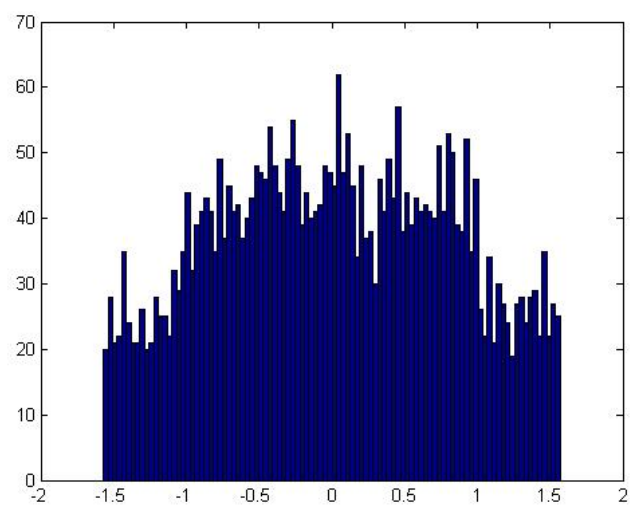

Fig 12 TWII 